ARTÍCULOS ORIGINALES Rev Chil Salud Pública 2015; Vol 19 (2): 130-139
Francisca Vidal Centro de Sistemas Públicos del Departamento de Ingeniería Industrial de la Universidad de Chile. fvidal@dii.uchile.cl

Eduardo Contreras Departamento de Ingeniería Industrial, Universidad de Chile.

Sara Arancibia Instituto de Ciencias Básicas, Facultad de Ingeniería Universidad Diego Portales.

ANTONIO INFANTE Centro de Sistemas Públicos del Departamento de Ingeniería Industrial de la Universidad de Chile.

\section{HETEROGENEIDAD EN LOS RESULTADOS DE GESTIÓN DE CENTROS DE SALUD FAMILIAR DE LA REGIÓN METROPOLITANA MEDIANTE UN MÉTODO MULTICRITERIO*}

\author{
Heterogeneity in management Results of PRimary CARE CENTERS \\ OF the Metropolitan Region using a MUlti-CRIteria MEthod
}

\section{RESUMEN}

Introducción: En Chile, la mayoría de los establecimientos de atención primaria dependen de los municipios, que cuentan con capacidades muy beterogéneas, lo cual se reproduce en la gestión de salud municipal. El objetivo del trabajo es presentar un índice de resultados de gestión de Centros de Salud Familiar (CESFAM), con el que se estudiará la beterogeneidad en la región Metropolitana.

Metodología: Siguiendo la metodología multicriterio y el proceso de análisis jerárquico, se define junto a un equipo de expertos una estructura jerárquica para evaluar los resultados de gestión de los CESFAM. Se determinan tres criterios estratégicos (gestión clinica, gestión administrativa y trabajo intersectorial), que por medio de comparaciones a pares obtienen ponderadores de $53,7 \%, 34,3 \%$ y $12,0 \%$, respectivamente. El indice es evaluado a través de 25 indicadores, utilizando información ya recopilada por los CESFAM.

Resultados: Se calculó el indice para 90 CESFAM, con datos del año 2013 disponibles en DEIS. Los CESFAM estudiados obtuvieron puntajes entre 0,26 y 0,81 (media 0,56, desviación estándar 0,12). Al realizar una categorización y una georreferenciación de los establecimientos se observa heterogeneidad en los resultados, la cual se manifiesta a nivel de red asistencial y comunal.

Discusión: Existen grandes diferencias en los resultados de gestión de los CESFAM, incluso entre establecimientos cercanos. Llama la atención la variabilidad al interior de algunas comunas, que no puede ser explicada por diferencias de recursos. Se identifica así un espacio importante de mejora, que puede ser abordado a través de actividades de colaboración entre establecimientos.

Palabras clave: atención primaria, multicriterio, heterogeneidad.

Recibido 12 de enero 2014, aceptado 28 de abril 2015

* Los autores agradecen el apoyo financiero del proyecto "Sistema de Gestión del Conocimiento para la Transferencia de Innovaciones y Buenas Prácticas de Gestión entre Gobiernos Locales", D10I 1034, provisto por el "XVIII Concurso de Proyectos de Investigación y Desarrollo" del Fondo de Fomento Científico y Tecnológico (FONDEF) de la Comisión Nacional de Ciencia y Tecnología (CONICYT). Igualmente, agradecen la colaboración de los directivos de Centros de Salud Familiar, que ha sido vital para el desarrollo de este trabajo. 


\begin{abstract}
Introduction: In Chile, most primary care centers are managed by municipalities, which have different capabilities. The aim of this work is to present an index to measure management results in primary care centers. This tool will be used to evaluate differences between primary care centers in the Metropolitan Region of Chile.

Method: Using a multi-criteria method and the Analytical Hierarchy Process, a bierarchical structure was defined by a group of experts, to measure management results in primary care centers. Three strategic criteria were obtained and then prioritized through pairwise comparisons, resulting in: Clinical management (53.7\%), Administrative management (34.3\%) and Intersectoral work $(12.0 \%)$. The index was built with 25 indicators that use information gathered by bealth centers for other purposes.

Results: Results were obtained for 90 primary care centers using data from 2013. Scores ranged between 0.26 and 0.81 (mean 0.56, standard deviation: 0.12). Through categorization and georeferential analysis, heterogeneity was observed, even at a neighborbood level.

Discussion: There are big differences in management results between primary care centers, even among nearby facilities. One issue that stands out is the beterogeneity found in centers managed by the same municipality, which cannot be explained by differences in budget. This is an opportunity for improvement that could be approached through collaborative work between facilities.
\end{abstract}

Key words: primary care, multi-criteria, heterogeneity.

\title{
INTRODUCCIÓN
}

La atención primaria (APS) es fundamental en los sistemas de salud, y los países que cuentan con un modelo de atención primaria robusto presentan mejores resultados de salud en su población, junto a mayor equidad y eficiencia en su provisión. ${ }^{1}$ En Chile, desde el retorno de la democracia la APS ha tomado un rol central, lo cual ha sido acompañado por la implementación del modelo de salud familiar. En el transcurso de los últimos años ha aumentado el número de establecimientos de atención primaria, su financiamiento, ${ }^{2}$ y su importancia dentro de las reformas y lineamientos del Ministerio de Salud. ${ }^{3}$

Sin embargo, la mayoría de los establecimientos de atención primaria en Chile dependen de los municipios, que presentan gran heterogeneidad respecto del tamaño de su población, sus recursos, su capacidad de gestión y las necesidades de salud de su población. ${ }^{4}$ Por ejemplo, el presupuesto de salud del año 2013 por inscrito según datos del SINIM $^{(i)}$ muestra diferencias de hasta un $490 \%$ entre municipios de la región Metropolitana.

Aspectos como éste generan una gran variabilidad en la gestión de salud municipal, lo cual ha sido abordado en distintos estudios, ${ }^{5,6}$ principalmente en forma cualitativa. Otros estudios cuantitativos analizan la gestión de establecimientos de salud por medio de su calidad percibida, ${ }^{7}$ se enfocan en el nivel secundario de atención ${ }^{8}$ o analizan resultados a nivel agregado de la red asistencial, ${ }^{9,10}$ no a nivel de establecimientos de atención primaria.

El presente estudio propone un índice de resultados de gestión de los CESFAM, construido utilizando la metodología multicriterio AHP, que será utilizado con el objetivo de estudiar la heterogeneidad en los resultados de los CESFAM de la región Metropolitana, con vistas a facilitar la colaboración y el aprendizaje entre CESFAM, en la medida que se establecen ámbitos de la gestión en los que los distintos centros pueden ser más fuertes o más débiles en términos relativos.

(i) Sistema Nacional de Información Municipal de la Subsecretaría de Desarrollo Regional y Administrativo (SUBDERE). 


\section{METODOLOGÍA}

Para la construcción del índice de resultados de gestión de los CESFAM se utilizó la metodología multicriterio y en particular el proceso de análisis jerárquico (Analytic Hierarchy Process, AHP), desarrollado por Thomas Saaty en 1970. Esta metodología fue escogida ya que permite la agregación de variables que se miden en distintas escalas y la incorporación de juicios de los tomadores de decisiones, asegurando su coherencia por medio del cálculo de un indicador de consistencia. ${ }^{11}$ Además, el AHP ha sido utilizado ampliamente en el sector salud, especialmente en estudios de evaluación en salud y políticas. ${ }^{12}$

El objetivo del modelo presentado es evaluar los resultados de gestión de un CESFAM en su población a cargo. Para la definición de resultados deseados se consideran los lineamientos establecidos por el MINSAL ${ }^{13-15}$ y la población a cargo corresponde a aquélla inscrita en el CESFAM, de acuerdo a los datos validados por FONASA.

El equipo de expertos fue seleccionado de acuerdo a un muestreo no probabilístico por criterio. Se escogió a seis directores de centros de salud, junto al director de un Servicio de Salud, todos de la región Metropolitana. De esta forma, se puede contrastar la visión de quienes están a cargo de la gestión de un CESFAM y conocen los desafíos a los que se enfrentan día a día, con la del director del servicio de salud, que entrega los lineamientos para el funcionamiento de los centros de atención primaria, conectando al MINSAL con la realidad de las localidades.

La estructura jerárquica a través de la cual se evalúan los resultados de gestión de los CESFAM se definió mediante una revisión bibliográfica y reuniones con el equipo de expertos. Se consensuó que los resultados de gestión de un CESFAM deben ser medidos por medio de tres criterios estratégicos: gestión clínica, gestión administrativa y trabajo intersectorial. De cada uno de ellos se des- prenden subcriterios y criterios terminales, que son evaluados con 25 indicadores.

Para abarcar un gran número de centros de salud se definió como alcance del modelo que los indicadores provengan de fuentes secundarias, principalmente DEIS ${ }^{(\mathrm{ii})}$ y datos de FONASA. Esta limitante provocó que algunos de los criterios se encuentren ajustados y que otros no pudieran ser considerados. Cabe destacar que en DEIS existen más de 185 planillas de información provista por los CESFAM, que se utiliza para evaluar metas sanitarias, indicadores de actividad y resultados de programas específicos.

La Tabla 1 muestra una definición de los criterios estratégicos incluidos en el modelo multicriterio y los indicadores utilizados para evaluar cada uno de ellos. Se generaron funciones de transformación para agregar los indicadores que se encontraban en distintas escalas, basados en un estudio de los resultados nacionales de los mismos para el año 2013 y en consenso con el equipo de expertos. De esta forma, el puntaje de cada indicador se encuentra entre 0 y 1 , siendo 1 el puntaje máximo.

Los ponderadores de los criterios fueron definidos en concordancia con la metodología AHP, efectuando comparaciones a pares en cada nivel del modelo. Los juicios de los expertos fueron combinados por medio de la media geométrica y se calculó el vector propio de la matriz de juicios, que sintetiza la escala de proporciones. ${ }^{\text {(iii) }}$

Las ponderaciones se presentan en la Figura 1. El criterio estratégico con mayor importancia relativa fue la gestión clínica, con un $53,7 \%$, seguido de gestión administrativa $(34,3 \%)$ y trabajo intersectorial $(12,7 \%)$.

Para la aplicación del índice se definió como universo a todos los establecimientos de atención primaria de la región Metropolitana, con los que se estudiaría la heterogeneidad de resultados utilizando datos del

\footnotetext{
(ii) Departamento de Estadísticas e Información en Salud.

(iii) Junto a esto, se calculó el valor propio de cada matriz, que entrega una medición de la inconsistencia de los juicios. De acuerdo a la metodología de Saaty, el máximo nivel de inconsistencia aceptado es $10 \%$. En este caso el mayor valor obtenido fue de $0,8 \%$.
} 


\section{Tabla 1. Criterios estratégicos e indicadores del Índice de Resultados de Gestión en CESFAM}

1. Gestión Clínica. Es el uso del conocimiento clínico para optimizar el proceso asistencial, para proporcionar el servicio requerido y para gestionar el tiempo asistencial. Incluye la gestión de la asistencia sanitaria y de los procesos asistenciales, que buscan mejorar el diagnóstico, tratamiento y cuidado de los pacientes, además de los resultados de la práctica clínica. ${ }^{16}$

\begin{tabular}{ll}
\hline Criterio & Indicador \\
\hline 1.1 Resolutividad & Tasa de interconsultas sobre consultas de morbilidad y de especialidades.
\end{tabular}

1.2 Atención en Programas

1.2.1 Programa infantil

1.2.2 Programa adolescente

1.2.3 Programa adulto

1.2.4 Programa adulto mayor

Cobertura hasta los 5 años 11 meses.

Recuperación del desarrollo psicomotor.

Población bajo control con obesidad, entre 0 y 6 años.

Altas odontológicas para las edades de 2, 4 y 6 años.

Cobertura de controles del joven sano.

Ingresos de gestantes menores de 15 años antes de las 14 semanas.

Altas odontológicas a los 12 años.

Cobertura de exámenes de medicina preventiva del adulto, para hombres entre 20 y 44 años y mujeres entre 45 y 64 .

Cobertura de prueba de Papanicolaou (PAP) en mujeres de 25 a 64 años.

Compensación efectiva de diabetes mellitus tipo II.

Compensación efectiva de hipertensión arterial.

Altas odontológicas para gestantes de 20 años y más.

Cobertura de exámenes de medicina preventiva del adulto mayor.

Número promedio de visitas domiciliarias integrales a pacientes con dependencia severa.

Compensación efectiva de diabetes mellitus tipo II.

Compensación efectiva de hipertensión arterial.

2. Gestión Administrativa. Refiere a las labores de carácter no clínico dentro de un CESFAM, como el manejo de los recursos (humanos, financieros, materiales, tecnológicos) y al conocimiento de la población a cargo, los que sirven de apoyo a la gestión clínica de un Centro de Salud Familiar. Por falta de información secundaria disponible no será posible incluir la evaluación de recursos humanos.

\begin{tabular}{ll}
\hline Criterio & Indicador \\
\hline 2.1 Disponibilidad de fármacos & $\begin{array}{l}\text { Porcentaje de recetas despachadas incompletas, respecto al total de recetas des- } \\
\text { pachadas. }\end{array}$ \\
2.2 Gestión de reclamos & $\begin{array}{l}\text { Porcentaje de reclamos respondidos dentro del plazo legal. } \\
\text { 2.3 Diagnóstico de familias a cargo }\end{array}$ \\
\hline
\end{tabular}

3. Trabajo Intersectorial. Consiste en la coordinación, con otros sectores sociales y gubernamentales, para potenciar la educación, prevención y fomento de salud, junto a la participación de la comunidad en las decisiones y la planificación del centro de salud.

\begin{tabular}{ll}
\hline Criterio & Indicador \\
\hline 3.1 Promoción & Número de eventos masivos de promoción. \\
3.2 Participación & Número de consejos consultivos, de desarrollo y comités locales. \\
\hline
\end{tabular}


Figura 1. Estructura Jerárquica del Índice de Resultados de Gestión en CESFAM

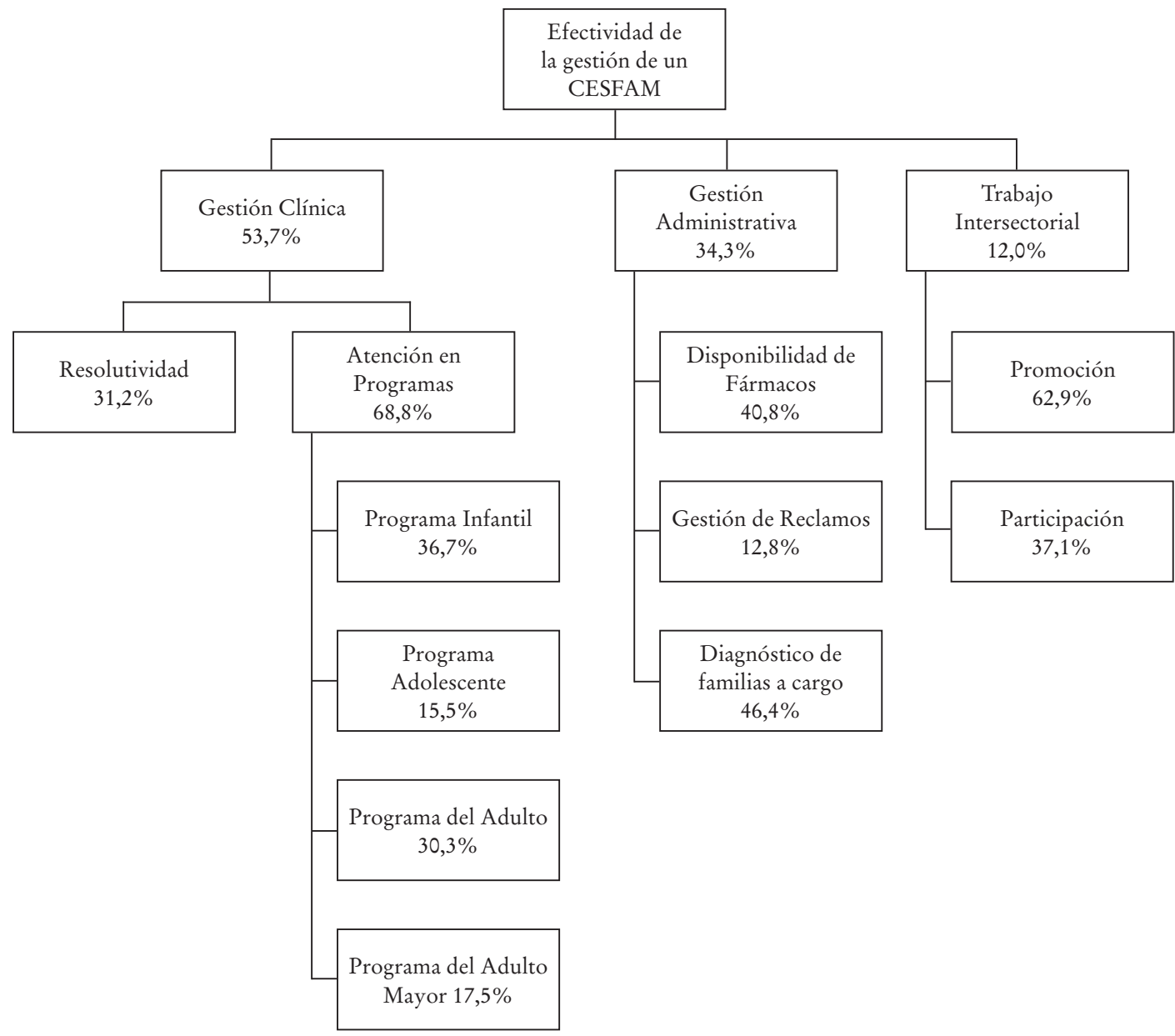

año 2013. Sin embargo, en DEIS no se enmétodo exige que se determine a priori el núcuentran los datos necesarios para la obtención del índice en todos los establecimientos, por lo que se debió acotar la muestra a 90 CESFAM de la región, seleccionados según un muestreo no probabilístico, ${ }^{17(i v)}$ en base a la disponibilidad de sus datos.

Con el índice ya calculado, se determinó una clasificación de los centros de salud de acuerdo a los resultados obtenidos. Para esto se utiliza la técnica de k-medias, que minimiza la distancia de cada observación a la media del conglomerado al cual pertenece. 18 Este mero de conglomerados a utilizar, que en este caso fueron tres, para clasificar a los CESFAM según puntajes altos, medios y bajos.

\section{RESULTADOS}

Se calculó el índice de resultados de gestión de CESFAM para 90 establecimientos de salud de la región Metropolitana, correspondientes al 56\% del total de la región, utilizando datos del año 2013 disponibles en DEIS.

Los CESFAM estudiados obtuvieron

(iv) Un muestreo no probabilístico corresponde a una técnica donde las muestras se recogen en un proceso que no brinda a todos los individuos de la población iguales oportunidades de ser seleccionados. ${ }^{17}$ 
puntajes entre 0,26 y 0,81 , con una media de 0,56 y una desviación estándar de 0,12. De los criterios estratégicos, el que obtuvo mayor media fue gestión administrativa $(0,58)$, seguido de gestión clínica $(0,57)$ y finalmente trabajo intersectorial $(0,48)$.

Junto a esto, se realizó una clasificación de los CESFAM según su puntaje final en el índice de resultados de gestión utilizando la técnica de k-medias. Se identificaron tres conglomerados: 1 . puntaje alto (media 0.72, 22 CESFAM), 2. puntaje medio (media 0.57, 37 CESFAM) y 3. puntaje bajo (media 0.44 , 31 CESFAM).

En la Figura 2 se presentan los CESFAM clasificados y desplegados en el mapa de la región Metropolitana, en el que se puede apreciar la diversidad de resultados. Se destaca la presencia de CESFAM de altos puntajes muy cercanos a otros de bajos puntajes, lo cual se repite en distintas zonas de la ciudad.

Figura 2: Mapa con resultados del Índice de Resultados de Gestión en CESFAM de la región Metropolitana

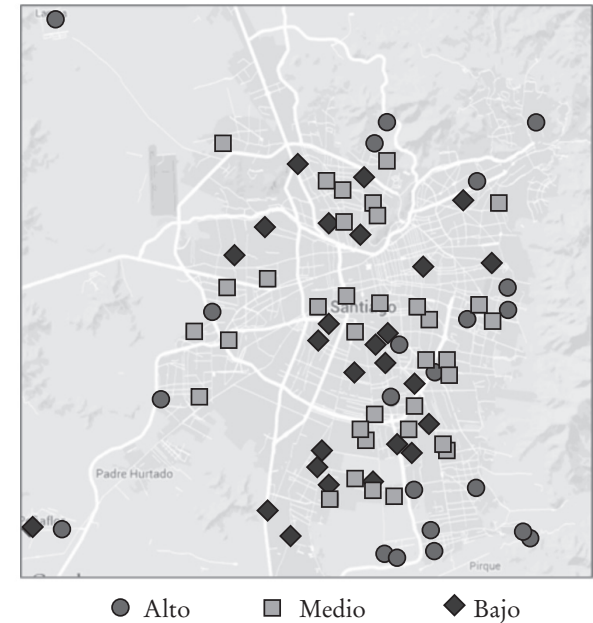

Al clasificar a los CESFAM de acuerdo a sus puntajes por criterio estratégico utilizando nuevamente la técnica de k-medias, se repite el fenómeno de la heterogeneidad entre CESFAM cercanos, tal como se presenta en la Figura 3. De los tres criterios estratégicos, la heterogeneidad es más intensiva en Trabajo Intersectorial, que cuenta con un coeficiente de variación de $73 \%$.
El detalle de la media de cada grupo, los puntajes de corte y el número de CESFAM pertenecientes a cada nivel se presentan en la Tabla 2.

Tabla 2. Caracterización de categorías según puntaje (Alto, Medio, Bajo), para Índice final y criterios estratégicos

\begin{tabular}{|c|c|c|c|c|}
\hline Criterio & & Alto & Medio & Bajo \\
\hline \multirow[t]{3}{*}{ Índice final } & Media & 0,72 & 0,57 & 0,44 \\
\hline & Puntaje de corte & 0,65 & 0,51 & - \\
\hline & $\begin{array}{l}\mathrm{N}^{\circ} \text { de } \\
\text { CESFAM } \\
\text { (Porcentaje } \\
\text { del total) }\end{array}$ & $\begin{array}{l}22 \\
(24,4 \%)\end{array}$ & $\begin{array}{l}37 \\
(41,1 \%)\end{array}$ & $\begin{array}{l}31 \\
(34,4 \%)\end{array}$ \\
\hline \multirow{3}{*}{$\begin{array}{l}\text { Criterio } \\
\text { estratégico: } \\
\text { Gestión } \\
\text { Clínica }\end{array}$} & Media & 0,68 & 0,55 & 0,37 \\
\hline & Puntaje de corte & 0,65 & 0,51 & - \\
\hline & $\begin{array}{l}\mathrm{N}^{\circ} \text { de } \\
\text { CESFAM } \\
\text { (Porcentaje } \\
\text { del total) }\end{array}$ & $\begin{array}{l}37 \\
(41 \%)\end{array}$ & $\begin{array}{l}17 \\
(19 \%)\end{array}$ & $\begin{array}{l}36 \\
(40 \%)\end{array}$ \\
\hline \multirow{3}{*}{$\begin{array}{l}\text { Criterio } \\
\text { estratégico: } \\
\text { Gestión } \\
\text { Administra- } \\
\text { tiva }\end{array}$} & Media & 0,92 & 0,54 & 0,34 \\
\hline & Puntaje de corte & 0,6 & 0,43 & - \\
\hline & $\begin{array}{l}\mathrm{N}^{\circ} \text { de } \\
\text { CESFAM } \\
\text { (Porcentaje } \\
\text { del total) }\end{array}$ & $\begin{array}{l}17 \\
(19 \%)\end{array}$ & $\begin{array}{l}60 \\
(67 \%)\end{array}$ & $\begin{array}{l}13 \\
(14 \%)\end{array}$ \\
\hline \multirow{3}{*}{$\begin{array}{l}\text { Criterio } \\
\text { estratégico: } \\
\text { Trabajo } \\
\text { Intersectorial }\end{array}$} & Media & 0,93 & 0,51 & 0,11 \\
\hline & Puntaje de corte & 0,71 & 0,3 & - \\
\hline & $\begin{array}{l}\mathrm{N}^{\circ} \text { de } \\
\text { CESFAM } \\
\text { (Porcentaje } \\
\text { del total) }\end{array}$ & $\begin{array}{l}27 \\
(30 \%)\end{array}$ & $\begin{array}{l}29 \\
(32 \%)\end{array}$ & $\begin{array}{l}34 \\
(38 \%)\end{array}$ \\
\hline
\end{tabular}


Figura 3. Mapa con resultados del Índice de Resultados de Gestión en CESFAM de la región Metropolitana, detallados por criterio estratégico
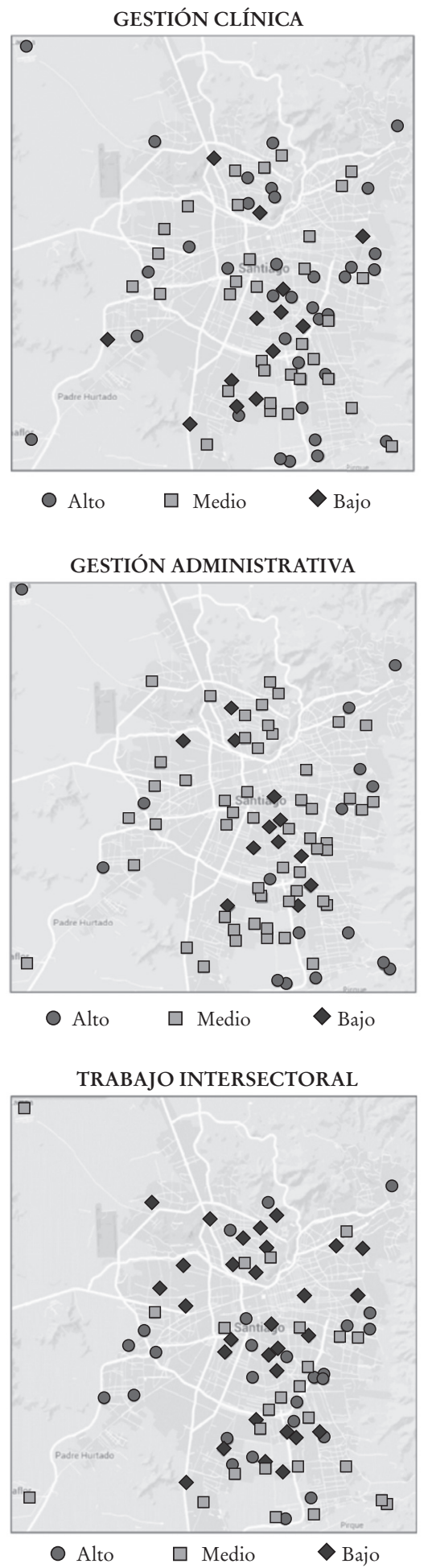

Figura 4. Detalle de resultados de CESFAM para una muestra de 12 comunas

\begin{tabular}{|c|c|c|c|c|c|c|c|}
\hline \multirow{2}{*}{$\begin{array}{l}\text { Comuna } \\
\text { Comuna } 1\end{array}$} & \multicolumn{7}{|c|}{ Clasificación CESFAM por puntaje final } \\
\hline & $\diamond$ & $\square$ & ○ & & & & \\
\hline Comuna 2 & $\diamond$ & $\diamond$ & $\diamond$ & $\square$ & $\square$ & $\square$ & ○ \\
\hline Comuna 3 & $\square$ & ○ & & & & & \\
\hline Comuna 4 & $\diamond$ & $\diamond$ & $\square$ & $\square$ & $\square$ & & \\
\hline Comuna 5 & $\diamond$ & ○ & & & & & \\
\hline Comuna 6 & $\square$ & ○ & & & & & \\
\hline Comuna 7 & $\square$ & $\square$ & ○ & & & & \\
\hline Comuna 8 & $\square$ & $\square$ & $\square$ & 0 & ○ & & \\
\hline Comuna 9 & $\diamond$ & ○ & & & & & \\
\hline Comuna 10 & $\square$ & $\square$ & $\square$ & ○ & 0 & & \\
\hline Comuna 11 & $\diamond$ & $\diamond$ & $\square$ & $\square$ & & & \\
\hline Comuna 12 & $\diamond$ & 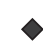 & $\square$ & & & & \\
\hline
\end{tabular}

Llama la atención que también a nivel comunal existe heterogeneidad. En la Figura 4 se presenta una muestra de 12 comunas en las cuales sus establecimientos muestran resultados dispares. Incluso en cuatro de ellas se encuentran CESFAM de altos y bajos puntajes, dependientes de una misma administración comunal.

Finalmente, a un nivel agregado de todos los CESFAM evaluados, los criterios terminales con mejores resultados fueron "Gestión de Farmacias" (puntaje promedio de 0,96) y "Gestión de Reclamos" (puntaje promedio de $0,75)$. Por otro lado, los criterios con puntajes más bajos fueron "Diagnóstico de Familias" (puntaje promedio de 0,208) y "Programa del Adolescente” (puntaje promedio de 0,407).

\section{DISCUSIÓN}

Del estudio presentado se desprende que existen grandes diferencias en los resultados de gestión de los CESFAM, lo que se traduce en una oportunidad importante para potenciar el trabajo en conjunto. 
Por ejemplo, los puntajes obtenidos en resolutividad -relacionada con la interacción del CESFAM con los hospitales o niveles superiores de atención y la capacidad del establecimiento de entregar respuesta a los problemas de su población- presentaron gran variabilidad entre los centros de un mismo Servicio de Salud, llegando a encontrarse coeficientes de variación de $48 \%$. Más aún, dentro de una misma red asistencial hay CESFAM con resultados en resolutividad sobre 0,8 y otros bajo 0,2. Éste es un indicio de que los Servicios de Salud aún tienen espacios para fomentar la integración de la red, motivando que los CESFAM se conozcan y compartan sus experiencias, otorgándole un rol primordial a los Consejos Integradores de la Red (CIRA) u otras herramientas que persigan el mismo fin.

No obstante, la integración no es una tarea exclusiva del Servicio de Salud. Como se vio anteriormente, las comunas también tienen resultados heterogéneos y pueden motivar el trabajo colaborativo de sus centros. Las fronteras del territorio tampoco deberían ser un obstáculo, ya que existen experiencias que buscan potenciar la gestión del conocimiento en APS a través de las tecnologías de información y otros medios de difusión. (v)

Un ejemplo de esto es el proyecto Colaboración Pública, que por medio de la cocreación y el design thinking busca materializar innovaciones en CESFAM, con diálogo y cooperación entre instituciones. La iniciativa ha logrado resultados concretos, como el aumento de la tasa promedio de atenciones para pacientes hipertensos y diabéticos en un $30,7 \%$ y un $39 \%$ respectivamente, en un centro de salud de la ciudad de Santiago, manteniendo los niveles de compensación de su población a cargo y con la misma dotación. Para más detalles se recomienda ver "Cocreación para la Innovación: Un caso en el Sector Público Chileno". ${ }^{19}$
Junto con la medición de la heterogeneidad, la herramienta presentada puede ser útil para determinar qué aspectos necesitan ser abordados con mayor urgencia a través de las metodologías colaborativas presentadas, al entregar una medición cuantitativa de las principales debilidades y fortalezas de un CESFAM. A nivel agregado, se encontró que las principales falencias están en "Diagnóstico de familias" y "Programa del Adolescente", por lo que se podría comenzar trabajando en estos dos desafíos.

Los hallazgos anteriores relativos a heterogeneidad de los resultados de gestión dentro de la región Metropolitana no deberían sorprender cuando se trata de diferencias entre comunas, pues en esos casos la hipótesis explicativa podría ser las diferencias en ingresos. Lo que más llama la atención son las importantes diferencias al interior de algunas de las comunas, donde surge como una hipótesis más atractiva la diferencia en las capacidades de gestión. Esto último estaría indicando un espacio importante de mejora a través de actividades de cooperación como las ya mencionadas.

A pesar de lo anterior, se debe tener en cuenta que existen algunas comunas mixtas en la región Metropolitana, que presentan realidades socioeconómicas y características de su población diferentes dentro de un mismo territorio, lo cual no estaría siendo identificado en el estudio y corresponde a una limitación de la herramienta presentada. De todas formas, debido a la alta segregación presente en la región Metropolitana, ${ }^{20}$ casos como éstos no son mayoritarios.

Futuros estudios podrían corregir limitaciones como la mencionada anteriormente, al controlar por factores externos que influyen en las condiciones de salud de la población, como nivel educacional e ingresos, prevalencia de ciertas enfermedades, distribución demográfica, entre otras.

(v) Algunos ejemplos son la Comunidad de Prácticas en APS (http://buenaspracticasaps.cl/) o los Concursos de Buenas Prácticas en Atención Primaria de Salud Municipal organizados por la Asociación Chilena de Municipalidades. 


\section{REFERENCIAS}

1. Starfield B, Shi L. Policy relevant determinants of health: an international perspective. Health Policy. 2002; 60(3): 201-218.

2. Centro de Políticas Públicas UC. Fortalecimiento de la atención primaria de salud: propuestas para mejorar el sistema sanitario chileno [en línea]. Serie Temas de la Agenda Pública; 2014 [consultado 21 nov 2014]. Disponible en: http://buenaspracticasaps.cl/wp-content/uploads/2014/05/ Pol\% C3\% ADticas-P\% C3\% B AblicasUC-2014-APS.pdf

3. Chile. Ministerio de Salud de Chile. Cuenta Pública 2010-2014 [en línea]; 2014 [consultado 10.01.2015] Disponible en: http:// web.minsal.cl/sites/default/files/Libro_ CP_2010-2014.pdf

4. Montero J, Téllez Á, Herrera C. Reforma sanitaria chilena y la atención primaria de salud. Algunos aspectos críticos [en línea] Serie Temas de la Agenda Pública; 2010 [consultado 10.8.2014]. Disponible en: http://politicaspublicas.uc.cl/wp-content/ uploads/2015/02/reforma-sanitaria-chilenay-la-atencion-primaria-de-salud.pdf

5. Asociación Chilena de Municipalidades. Estudio Diagnóstico de la Situación de Gestión de Salud en las Entidades Administradoras e Identificación de Buenas Prácticas Locales [en línea]. Santiago: Alcalá Consultores Asociados Limitada; 2009 [consultado 12.08.2014]. Disponible en: http://www. munitel.cl/file_admin/archivos_munitel/salud/salud38.pdf

6. Pontificia Universidad Católica de Chile, Consorcio Universitario en Salud y Medicina Familiar Asociación de Facultades de Medicina en Chile (ASOFAMECH). Análisis diagnóstico de la gestión municipal en entidades administradoras de Salud. Informe Final. 2009.

7. Chile. Superintendencia de Salud. Satisfacción y Calidad Percibida en la Atención de Salud Hospitalaria: Ranking de Prestadores [en línea]. Chile: Departamento de Estudios y Desarrollo; 2013 [consultado 10.04.2015]. Disponible en: http://www.supersalud.gob.cl/documentacion/569/articles-9005_recurso_1.pdf
8. Farga A. Los mejores en 3D. América Economía Intelligence [en línea]; sept 2011. [consultado 15.04.2015]. Disponible en: http:// www.uchile.cl/documentos/ranking-de-clinicas-y-hospitales-2011_75447_0_3917.pdf

9. Subsecretaría de Redes Asistenciales. Balance de Gestión Integral 2013 [en línea] Chile: MINSAL; 2013 [consultado 12.04.2015]. Disponible en: http://www.dipres.gob. cl/595/articles-114846_doc_pdf.pdf

10. Servicios de Salud. Balance de Gestión Integral 2013. [en línea] Chile: Ministerio de Salud; 2013 [consultado 12.04.2015] Disponible en: http://www.dipres.gob.cl/595/articles-114842_doc_pdf.pdf

11. Saaty T. Toma de Decisiones para Lideres: Universidad de Santiago; 1997.

12. Liberatore M, Nydick R. The analytic hierarchy process in medical and health care decision making: a literature review. Eur J Oper Res. 2008; 189(1): 194-207.

13. Chile. Ministerio de Salud. Orientaciones para la Planificación y Programación en Red [en línea]. 2013 [consultado 14.04.2014]. Disponible en: http://web.minsal.cl/portal/url/item/ e6485ed1642ee853e040010164011633.pdf

14. Chile. Ministerio de Salud de Chile. Orientaciones para la implementación del modelo de atención integral de salud familiar y comunitaria [en línea] [consultado 8.05. 2014]. Disponible en: http://web. minsal.cl/portal/url/item/e7b24eef3e5cb5d1e0400101650128e9.pdf

15. Chile. Ministerio de Salud de Chile. Estrategia Nacional de Salud para el cumplimiento de los Objetivos Sanitarios de la Década 2011-2020 [en línea] [consultado 2.06.2014]. Disponible en: http://web. minsal.cl/portal/url/item/c4034eddbc96ca6de0400101640159b8.pdf

16. Román A. Basic concepts and definitions of clinical management. Medwave. 2012 Jun; 12(5): e5418 doi: 10.5867/medwave.2012.05.5418

17. Torres M, Paz K, Salazar F. Tamaño de una muestra para una investigación de mercado. Universidad Rafael Landívar: Boletín electrónico [en línea]. [consultado 6.04.2015] Disponible en: http://www.tec.url.edu.gt/ boletin/URL_02_BAS02.pdf 
18. Kanungo T, Mount D, Netanyahu N, Piatko C, Silverman R, Wu A. An Efficient k-Means Clustering Algorithm: Analysis and Implementation. [en línea] IEEE; 2002 [consultado 8.04.2015] Disponible en: https://www.cs.umd.edu/ mount/Projects/ KMeans/pami02.pdf

19. Waissbluth M, Contreras E, Galaz P, et al. Co-creación para la Innovación: Un caso en el Sector Público Chileno. Revista Ingenie- ría de Sistemas [en línea] 2014 [consultado 11.12.2014] Disponible en: http://www.dii. uchile.cl/ ris/RIS2014/1innovacion.pdf

20. Sabatini F, Wormald G, Salcedo R. Informe Final PBCT Anillos de Investigación en Ciencias Sociales. Barrios en Crisis y Barrios Exitosos producidos por la Política de Vivienda Social. Informe de resultados presentado a CONICYT. 2008. 